\title{
SYNTHESIS AND PROPERTIES OF HYDROXYETHYLCELLULOSE GRAFT COPOLYMERS AS SUPER WATER-ABSORBENTS
}

\author{
By Namiko Miyata ${ }^{* 1}$ and Isao Sakata ${ }^{* 2}$ \\ *1 Nakamura Gakuen College, Befu, Jonan-ku, Fukuoka, 814-01 Japan \\ *2 Faculty of Agriculture, Kyushu University, Hakozaki. Higashi-ku. Fukuoka, 812 Japan
}

\begin{abstract}
Graft copolymers of hydroxyethylcellulose (HEC) and other polysaccharides (PS) [e.g., mannan, alginic acid, agar, pectin and starch] containing partially hydrolyzed polyacrylamide (P-Hyd-PAM) or poly(methyl acrylate) (P-Hyd-PMA), poly(2-dimethyl aminoethyl methacrylate) (PDM) and poly (sodium acrylate) (PAA-Na) were synthesized as super water-absorbents. The absorbency of HEC-P-Hyd-PAM was greater than that of other polysaccharides and HEC graft copolymers containing the other types of branch polymers. The HEC -P-Hyd-PAM graft copolymers absorbed deionized water and a physiological saline solution up to $1000-3000 \mathrm{~g} / \mathrm{g}$ and $100-300 \mathrm{~g} / \mathrm{g}$ of absorbent, respectively. These values were 3 - 5 times greater than those of commercially available super water-absorbents. The HEC and the monomers were converted to the trunk and branch polymers of the HEC graft copolymers in the yields of about $30-60 \%$ and $20-80 \%$, respectively. With an increase in grafting, the absorbencies of water and saline solutions of HEC-P-Hyd-PAM increased and reached maxima at $150-300 \%$ grafting. while those of HEC-PDM and the compressive strength of both gels increased monotonously. The gels of HEC-P-Hyd-PAM showed a discrete volume change at certain $\mathrm{CH}_{3} \mathrm{OH}-\mathrm{H}_{2} \mathrm{O}$ compositions. The absorbencies of water and saline solutions of the graft copolymers of $\mathrm{HEC}$ and the other polysaccharides depended on the viscosity of the trunk polymers.
\end{abstract}

\section{INTRODUCTION}

Since starch-acrylonitrile graft copolymers named "Super-Rubbers" were synthesized by Fanta et. al.[1], super water-absorbents have received considerable attention, and are now used as sanitary materials, agricultural and gardening agents, industrial dehydration agents, sensors and drying preven tives, etc. Three kinds of super water-absorbents. starchy, cellulosic and synthetic polymeric materials are currently known. However, the water absorbencies of the cellulosic super water-absorbents prepared from carboxymethylcellulose (CMC) and other cellulosic materials [2,3] are smaller than those of starchy and synthetic water-absorbents. The main disadvantage of most super water-absorbents is the difficulty in the absorption of ionized water such as a physiological saline solution $(0.85 \% \mathrm{NaCl}$ solution $)$ and urine[2].

In this study, an attempt was made to synthesize graft copolymers of HEC and other polysaccharides as super water-absorbents, which do not have the above mentioned disadvantage and are able to absorb much more ionized water. Some of their properties were investigated in comparison with those of commercial products. Some parts of this study were reported in a previous paper[4], and this paper de. scribes further details of the study.

\section{EXPERIMENTAL}

\subsection{Materials}

Hydroxyethylcelluloses (HEC) with the molar substitution (MS) of $1.2-4.0$ were reagent grade and used as received from Tokyo Chemical Industry $\mathrm{C}_{0}$. Ltd. and Daicel Chemical Industries, Ltd. Other poly. saccharides (konjac mannan, alginic acid, agar, starch and pectin) and $N, N$ '-methylenebisacrylamide were reagent grade and used as purchased from Tokyo Chemical Industry Co., Ltd. Acrylamide (AM), methyl acrylate (MA), acrylic acid (AA), 2-dimethylamino 
ethyl methacrylate (DM) and other chemicals were reagent grade and purified by conventional methods. Commercial super water-absorbents, i.e., SanwetIM1000 (starch-PAA), Sumika-gel NP-1010 [poly (vinvl alcohol) (PVA)-PAA] and GP-03 (PVA-maleic acid ester) were obtained from Sanyo Chemical Industries, Ltd. Sumitomo Chemical Industries, Ltd., and Nippon Synthetic Chemical Industry
Co., Ltd., respectively, and used without further purification.

\subsection{Synthesis}

Grafting of $A M$ and other monomers onto $H E C$ and other polysaccharides was carried out in the presence of $N, N^{*}$-methylenebisacrylamide as a bridging agent using ceric ammonium nitrate $\left(\mathrm{Ce}\left(\mathrm{NO}_{3}\right)_{4} \cdot 2 \mathrm{NH}_{4} \mathrm{NO}_{3}\right.$ : $8.3 \times 10^{-4} \mathrm{~mol} / l$ ) as an initiator [5] in a $0.06 \mathrm{~N}$

Table 1 Super Water-absorbents from HEC

\begin{tabular}{|c|c|c|c|c|c|c|c|c|c|}
\hline Materials & $\begin{array}{c}\text { Monomer } \\
\text { conc. } \\
(\%)\end{array}$ & $\begin{array}{c}\text { Trunk } \\
\text { polymer } \\
\text { conc. } \\
(\%)\end{array}$ & $\begin{array}{c}\text { Bridging } \\
\text { agent } \\
\text { conc. } \\
(\%)\end{array}$ & $\begin{array}{c}\text { Grafting } \\
\text { time } \\
\text { (h) }\end{array}$ & $\begin{array}{c}\text { Degree } \\
\text { of } \\
\text { grafting } \\
(\%)\end{array}$ & $\begin{array}{c}\text { Hydrolysis } \\
\text { time } \\
\text { (min) }\end{array}$ & $\begin{array}{c}\text { Degree } \\
\text { of } \\
\text { hydrolysis } \\
(\%)\end{array}$ & $\begin{array}{c}\text { Trunk } \\
\text { polymer/ } \\
\text { HEC } \\
(\%)\end{array}$ & $\begin{array}{c}\text { Branch } \\
\text { polymer/ } \\
\text { monomer } \\
(\%)\end{array}$ \\
\hline HEC-P-Hyd-PAM 1 & 2.5 & 2.5 & 0.3 & 2.0 & 98.0 & 20 & 89.3 & - & - \\
\hline HEC-P-Hyd-PAM 2 & 15.0 & 5.0 & 0.3 & 1.0 & 141.4 & 20 & 49.8 & 46.3 & 21.8 \\
\hline HEC-P-Hyd-PAM 3 & 5.0 & 2,5 & 0.3 & 5.0 & 161.7 & 84 & 52.9 & 38.3 & 30.8 \\
\hline HEC-P-Hyd-PAM 4 & 5.0 & 2.5 & 0.3 & 2.8 & 281.8 & 40 & 60.8 & 28.0 & 39.7 \\
\hline HEC-P-Hyd-PAM 5 & 15.0 & 5.0 & 0.3 & 2.0 & 424.8 & 20 & 63.9 & 37.5 & 53.1 \\
\hline HEC-P-Hyd-PAM 6 & 5.0 & 2.5 & 0.3 & 2.0 & 507.1 & 20 & 65.9 & 30.5 & 77.3 \\
\hline HEC-P-Hyd-PAM 7 & 12.5 & 2.5 & 0.3 & 2.0 & 615.8 & 5 & 41.4 & - & - \\
\hline HEC-P-Hyd-PAM 7 & 12.5 & 2.5 & 0.3 & 2.0 & 615.8 & 10 & 66.0 & - & - \\
\hline HEC-P-Hyd-PAM 7 & 12.5 & 2.5 & 0.3 & 2.0 & 615.8 & 20 & 74.6 & - & - \\
\hline HEC-P-Hyd-PAM 7 & 12.5 & 2.5 & 0.3 & 2.0 & 615.8 & 25 & 88.1 & - & - \\
\hline HEC-P-Hyd-PAM 8 & 15.0 & 5.0 & 0.1 & 3.5 & 373.2 & 20 & 57.2 & 38.8 & 48.2 \\
\hline HEC-P-Hyd-PAM 9 & 5.0 & 2.5 & 0.6 & 2.3 & 312.7 & 25 & 58.1 & 48.3 & 75.8 \\
\hline HEC-P-Hyd-PAM10 & 5.0 & 2.5 & 0.9 & 1.8 & 309.9 & 20 & 54.3 & 42.3 & 82.2 \\
\hline HEC-P-Hyd-PAM11 & 5.0 & 2.5 & 1.5 & 1.1 & 590.1 & 20 & 58.4 & 8.7 & 25.7 \\
\hline HEC-PDM 1 & 12.5 & 2.5 & 1.3 & 19.0 & 30.9 & - & - & 61.0 & 9.0 \\
\hline HEC-PDM 2 & 12.5 & 2.5 & 0.3 & 18.5 & 388.8 & - & - & - & - \\
\hline HEC-PDM 3 & 12.5 & 2.5 & 0.6 & 17.5 & 407.5 & - & - & - & - \\
\hline HEC-PDM 4 & 5.0 & 2.5 & 1.0 & 21.2 & 449.1 & - & - & - & - \\
\hline HEC-PDM 5 & 12.5 & 2.5 & 1.0 & 21.7 & 613.1 & - & - & 59.0 & 72.2 \\
\hline HEC-P-Hyd-PMA & 12.5 & 2.5 & 0.3 & 0.8 & - & 60 & 75.6 & - & - \\
\hline $\mathrm{HEC}-\mathrm{AA}$ & 5.0 & 2.5 & 0 & 16.2 & 73.0 & - & - & - & - \\
\hline HEC-PAANa & 5.0 & 2.5 & 0 & 2.8 & 392.5 & 30 & - & - & - \\
\hline
\end{tabular}

Table 2 Super Water-absorbents from Other Polysaccharides

\begin{tabular}{|c|c|c|c|c|c|c|c|c|c|}
\hline Materials & $\begin{array}{c}\text { Monomer } \\
\text { conc. } \\
(\%)\end{array}$ & $\begin{array}{c}\text { Trunk } \\
\text { polymer } \\
\text { conc } \\
(\%)\end{array}$ & $\begin{array}{c}\text { Bridging } \\
\text { agent } \\
\text { conc. } \\
(\%)\end{array}$ & $\begin{array}{l}\text { Grafting } \\
\text { time } \\
\text { (b) }\end{array}$ & $\begin{array}{c}\text { Degree } \\
\text { of } \\
\text { grafting } \\
(\%)\end{array}$ & $\begin{array}{c}\text { Hydrolysis } \\
\text { time } \\
\text { (min) }\end{array}$ & $\begin{array}{c}\text { Degree } \\
\text { of } \\
\text { hydrolysis } \\
(\%)\end{array}$ & $\begin{array}{c}\text { Trunk } \\
\text { polymer/ } \\
\text { PS } \\
(\%)\end{array}$ & $\begin{array}{c}\text { Branch } \\
\text { polymer/ } \\
\text { monomer } \\
(\%)\end{array}$ \\
\hline Mannan-P-Hyd-PAM & $12.5^{\circ}$ & 2.5 & 0.3 & 2.8 & 392.5 & 30 & 57.3 & - & - \\
\hline Alginic acid-P-Hyd-PAM & 12.5 & 2.5 & 0.3 & 16.0 & 219.8 & 30 & 55.1 & - & - \\
\hline Starch-P-Hyd-PAM & 12.5 & 2.5 & 0.3 & 1.5 & 184.3 & 20 & 68.2 & 78.1 & 36.7 \\
\hline Agar-P-Hyd-PAM & 12.5 & 2.5 & $=0.3$ & 4.8 & 169.4 & 20 & 55.3 & 50.7 & 17.2 \\
\hline Pectin-P-Hyd-PAM & 12.5 & 2.5 & 0.3 & 17.7 & 281.7 & 20 & 46.3 & 95.0 & 73.1 \\
\hline Sodium Arginiate-P-Hyd-PAM & $\mathrm{M} \quad 12.5$ & 2.5 & 0.3 & 21.5 & 253.5 & 20 & 57.7 & $一$ & - \\
\hline
\end{tabular}


$\mathrm{HNO}_{3}$ solution under nitrogen stream at $40{ }^{\circ} \mathrm{C}$ for a prescribed time. After grafting, homopolymers (PAM, PAA and PDM) and unreacted trunk polymers were removed by extraction with water for $1-2$ days, and PMA was removed by extraction with acetone for 1 day. Polyacrylamide and the other branch polymers which could not be removed by the extraction were supposed to be graft copolymerized.

The degree of grafting was determined as follows; the contents of PAM and PDM in the HEC graft copo. lymers were caluculated from the nitrogen contents determined by the Kjeldahl method, and the contents of PAA in HEC-PAA and HEC-PAA-Na were measured by a calcium acetate method.

The branch polymers, i.e. PAM and PMA in the graft copolymers of the HEC and the other polysaccharides were partially hydrolyzed with $0.5 \mathrm{~N}-\mathrm{NaOH}$ solutions at $100{ }^{\circ} \mathrm{C}$ for $20-100 \mathrm{~min}$. The products (HEC-P-Hyd-PAM and others) were neutralized with $\mathrm{HCl}$ solutions and precipitated with methanol. They were then dissolved in a diluted $\mathrm{NaOH}$ solution, precipitated again with methanol and dried in a vacuum for $48 \mathrm{~h}$ at $40^{\circ} \mathrm{C}$. The degree of hydrolysis of HEC-P-Hyd-PAM was determined from the nitrogen content, and the carboxyl groups content of HECP-Hyd-PMA was determined by the calcium acetate method. Tables 1 and 2 show the super waterabsorbents obtained from the HEC and the other polysaccharides, respectively.

\subsection{Fluid Absorbency Test}

About $0.1 \mathrm{~g}$ of each water-absorbent was put into $100-200 \mathrm{ml}$ of deionized water, $50 \mathrm{ml}$ of $0.85 \% \mathrm{NaCl}$ solution or $100 \mathrm{ml}$ of an aqueous methanol solution. After $16 \mathrm{~h}$. the swollen water-absorbents were separated from an unabsorbed solution by screening through an 80 mesh sieve. The unabsorbed solutions were then weighed, and the fluid absorbency was calculated as grams of absorbed solution per gram of absorbent.

The $\mathrm{pH}$ of the solutions was adjusted with $\mathrm{HCl}$ and $\mathrm{NaOH}$.

\subsection{Viscosity Determination}

The viscosities of the trunk polymers were measured with a rotating viscometer (Tokyo Keiki $C_{0}$.) at $20^{\circ} \mathrm{C}$.

\subsection{Compressive Strength Determination}

Compressive strengths of the gels of the waterabsorbents were determined with a Tom-200 type Universal Testing Machine at $20{ }^{\circ} \mathrm{C}$. The samples in the form of a cylinder, $29 \mathrm{~mm}$ in diameter, were compressed between flat parallel plates at a crosshead speed of $50 \mathrm{~mm} / \mathrm{min}$.

\section{RESULTS AND DISCUSSION}

\subsection{Synthesis and Fluid Absorbency}

Table 1 shows that HEC was converted to the trunk polymer in $30-60 \%$ yield, and the monomers were converted into the branch polymers in the HEC graft copolymers in $20-80 \%$ yield. The conversions of the monomers ( $\mathrm{AM}$ and $\mathrm{DM}$ ) to the branch polym. ers increased with an increase in the degree of graft. ing. However, the conversion of HEC to the trunk polymer was almost constant as shown in Fig. 1. These results suggest that in HEC the number of sites for grafting were limited and the monomers were polymerized on the grafting sites. They were converted to the branch polymers and only the degree of the polymerization increased. Fig. 1 shows that the HEC and the monomers were converted to the trunk polymer and the branch polymers in $40-50 \%$ and $75-$ $82 \%$ yields, respectively, in the presence of 0.3 $0.9 \%$ of the bridging agent. The yields were lower on $0.1 \%$ and $1.5 \%$ addition of the bridging agent.

Figs. 2 and 3 show the effects of the kinds of trunk

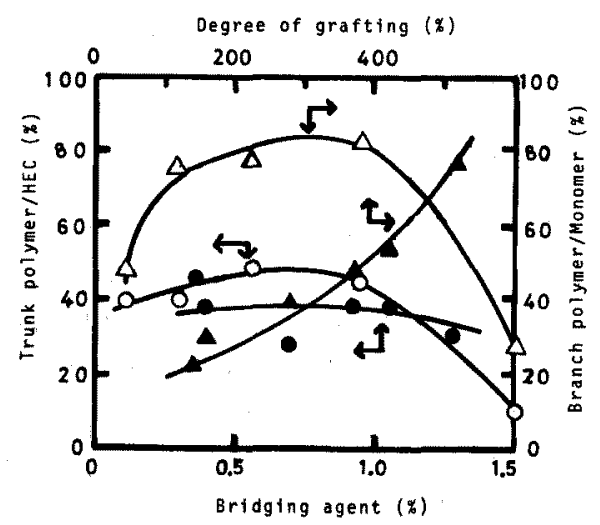

Fig. 1. Trunk polymer/HEC (\%) and branch polymer/ monomer (\%) versus bridging agent concentration and the degree of grafting of HEC-P-Hyd-PAM. Trunk polymer/HEC: $O$, bridging agent, degree of grafting. Branch polymer/Monomer: $\Delta$, bridging agent, $\Delta$, degree of grafting. 


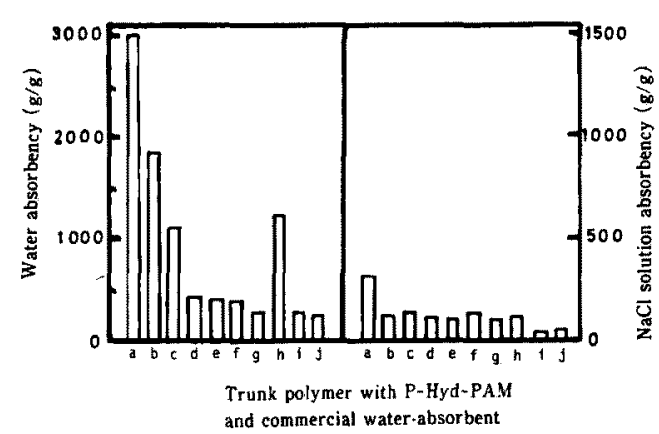

Fig. 2. Effects of the trunk polymers of the graft copolymers containing P-Hyd-PAM on the absorbencies of water and $0.85 \% \mathrm{NaCl}$ solution in comparison with commercial super water-absorbents. a, HEC; b, mannan; $c$, algiaic acid; $d$, starch; e, agar; $f$, pectin; $g$, sodium alginate; h. Sanwet; i, Sumika-gel; and j, GP.

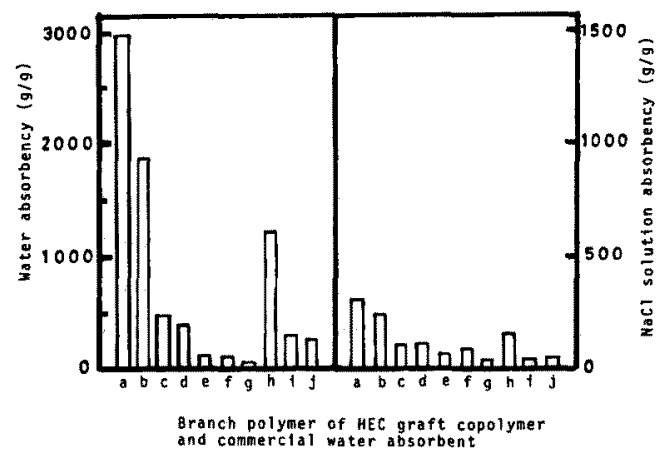

Fig. 3. Effects of the branch polymers of the HEC graft copolymers on the absorbencies of water and $0.85 \%$ $\mathrm{NaCl}$ solution in comparison with commercial super water-absorbents. a, P-Hyd-PAM; b, P-Hyd-PMA; c, PAA-Na; d, PDM; e, PAA; f, PAM; g. PMA; h, Sanwet; $i$, Sumika.gel; and j. GP.

and branch polymers on the absorbencies of distilled water and $0.85 \% \mathrm{NaCl}$ solution. The absorbencies of water and $0.85 \% \mathrm{NaCl}$ solution of the HEC graft copolymers were about three times as much as those of the graft copolymers of other polysaccharides and the commercial water-absorbents, and HEC-P-Hyd-. PAM could absorb $\mathrm{NaCl}$ solution and water up to 325 $\mathrm{g} / \mathrm{g}$ and $3000 \mathrm{~g} / \mathrm{g}$ of absorbent, respectively. The absorbencies of water and $0.85 \% \mathrm{NaCl}$ solution decreased in the oder, HEC $>$ konjac mannan, alginic acid $>$ starch $>$ agar $>$ pectin, and partially hydrolized $\mathrm{PAM}>$ partially hydrolized $\mathrm{PMA}>\mathrm{PAA}-\mathrm{Na}>$ $\mathrm{PDM}>\mathrm{PAA}>\mathrm{PAM}$, that is to say, partially hyd

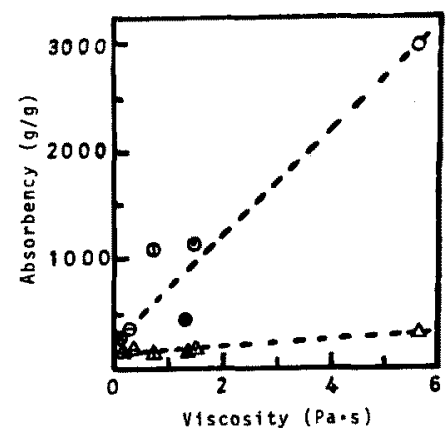

Fig. 4. Absorbencies of water and $0.85 \% \mathrm{NaCl}$ solution of the graft copolymers versus the viscosities of the trunk polymers. $\mathrm{H}_{2} \mathrm{O}: \mathrm{O}, \mathrm{HEC} ; \odot$, mannan; - starch; (1. alginic acid; $\Theta$. pectin; and $\Theta$, sodium alginate. $\mathrm{NaCl}$ soln.: $\triangle$. HEC; $\triangle$, mannan; $\Delta$. starch; and $\Delta$, alginic acid; $\Delta$, pectin; and $\Delta$, sodium alginate.

rolyzed $>$ ionic and hydrophilic $>$ hydrophilic $>$ hydrophobic, as shown in Figs. 2 and 3.

Fig. 4 shows that the viscosity of HEC was high and the HEC graft copolymers could absorb a lot of water. However, those of sodium alginate, pectin and alginic acid were low and their graft copolymers were unable to absorb a large quantity of water. It is concluded therefore that absorbencies of water and $\mathrm{NaCl}$ solution of the graft copolymers of HEC and other polysaccharides depended on the viscosity of the trunk polymer.

\subsection{Effects of Bridging Agent and Degree of Grafting}

The mechanism of water absorption of super waterabsorbents is different from that of cottons or sponges which is based on capillary action, and can be acounted based on the theory of the ionization of the polymer network $[6,7]$. The forces for the absorption of pure water and saline solution are attributed to the osmotic pressure based on the movable counter ions (e.g. $\left.\mathrm{Na}^{+}\right)$, the polymer-solvent affinity and the rubber elasticity which relates to the bridging density of the super water-absorbents. The water absorbencies of super water-absorbents are determined by the balance of these forces. The water absorbencies and the strength of super water-absorbents can be expressed by Eq. 1 Flory's equation(6) and Eq. $2[8]$, respectively. 


$$
\begin{aligned}
& \mathrm{Q}^{3 / 5}= {\left[\left(\mathrm{i} / 2 \cdot \mathrm{VuS}^{1 / 2}\right)^{2}\right.} \\
&\left.+\left(1 / 2-\mathrm{X}_{1}\right) / \mathrm{V}_{1}\right] /\left(\nu_{\mathrm{e}} / \mathrm{Vo}_{\mathrm{o}}\right) \\
& \mathrm{G}=\mathrm{RT} \cdot \nu_{\mathrm{e}} / \mathrm{Vo}
\end{aligned}
$$

where $\mathrm{Q}$ is degree of swelling, $\mathrm{i} / \mathrm{Vu}$ is charge density of polymer, $S *$ is ionic strength of solution, $(1 / 2-$ $\left.\mathrm{X}_{1}\right) / \mathrm{V}_{1}$ is polymer-solvent affinity, $\nu_{\mathrm{e}} / \mathrm{V}_{0}$ is bridging density and $G$ is rigidity.

Their water absorbencies, therefore, increase with an increase in the number of hydrophilic and ionic functional groups, and with a decrease in the bridging density of the polymer network and in the concentration of ionic solvents ( $\mathrm{NaCl}$ solution, urine etc.).

We reported in the previous paper [4] that as the degree of hydrolysis of branch PAM increased, the water absorbency of HEC-P-Hyd-PAM increased while the compressive strengh decreased.

Fig. 5 shows that as the amount of bridging agents incresed, the absorbencies of water and $\mathrm{NaCl}$ solu. tions of HEC-P-Hyd-PAM decreased and those of HEC-PDM increased, while, the compressive strengths of the gels of HEC-P-Hyd-PAM and HEC-PDM also increased.

The absorbencies of water and $\mathrm{NaCl}$ solutions of HEC-P-Hyd-PAM were in good agreement with Eq. 1.

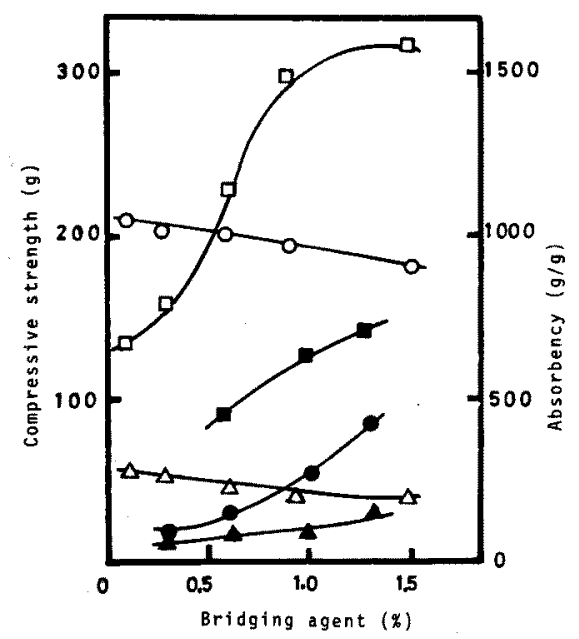

Fig. 5. Effects of bridging agent concentration on the compressive strength and absorbencies of water and $0.85 \% \mathrm{NaCl}$ solution of HEC-P-Hyd-PAM and HEC-PDM. HEC-P-Hyd-PAM: $O$, water absorbency; $\triangle$, $\mathrm{NaCl}$ absorbency; and $\square$, compressive strength. HEC-DM: - water absorbency; $\boldsymbol{A}, \mathrm{NaCl}$ absorbency; and $\square$, compressive strength.

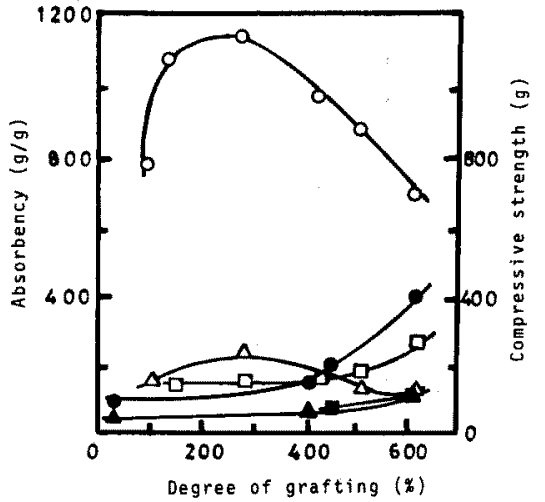

Fig. 6. Effects of the degree of grafting on absorbencies of water and $0.85 \% \mathrm{NaCl}$ solution and the compressive strength of HEC-P-Hyd-PAM and HEC-PDM. The symbols are the same as shown in Fig. 5.

while, those of HEC-PDM were in poor agreement. The compressive strength of HEC-P-Hyd-PAM and HEC-PDM agreed with Eq. 2 because their rigigity decreased as their bridging density increased.

Fig. 6 shows the effects of the degree of grafting on the absorbencies of water and $\mathrm{NaCl}$ solutions and the compressive strength. With an increase in the degree of grafting, the absorbencies of water and $\mathrm{NaCl}$ solutions of HEC-P-Hyd-PAM increased, reached maxima at $150-300 \%$ grafting and then gradually decreased. However, those of HEC-PDM increased monotonously with grafting. It is reported[ 3 ] that water and saline retention values (WRV and SRV) of the hydrolyzed polyacrylnitrile grafted cotton fibers increased with grafting and then leveled off at about $35 \mathrm{~g} / \mathrm{g}$ and 15 $\mathrm{g} / \mathrm{g}$, respectively, as the degree of grafting reached about $120-140 \%$. However, WRV of the DM-grafted fibers increased with grafting and was $7.0 \mathrm{~g} / \mathrm{g}$ at $110 \%$ grafting. Though the behavior is similar to that of HEC water-absorbents, these WRV and SRV values are much lower than those of HEC water-absorbents.

The compressive strength of the gels of the HECP-Hyd-PAM and HEC-PDM graft copolymers increased with an increase in the degree of grafting, and the absorbency and the compressive strength of the former was greater than those of the later as shown in Fig. 6. These results led to the conclusions that their water absorbencies increase with an increase in the number of hydrophilic and ionic func. 


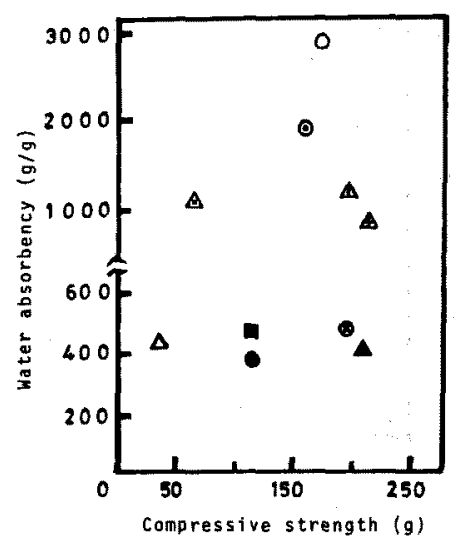

Fig. 7. Water absorbency versus the compressive strength of the graft copolymers of HEC and the other polysaccharides. $O$, HEC-P-Hyd-PAM: $\odot$, HEC-. P-Hyd-MA; $\otimes$, HEC-PAA-Na; O, HEC-P-DM; $\Delta$, alginic acid-P-Hyd-PAM; $\triangle$, mannan-P-Hyd-PAM; $\Delta$, sodium alginate-P-Hyd-PAM: $\triangle$, agar-P-Hyd-PAM; $\boldsymbol{\Delta}$. starch-P-Hyd-PAM; $\square$. commercial water-absorbent (Sanwet).

tional groups and in the osmotic pressure as mentioned above, and with a decrease in the bridging de. nsity.

Fig. 7 shows that the compressive strength of the HEC graft copolymers containing various branch polymers were close to each other from $110 \mathrm{~g}$ to $190 \mathrm{~g}$. although their water absorbencies were very different from $380 \mathrm{~g} / \mathrm{g}$ to $3000 \mathrm{~g} / \mathrm{g}$. However, the graft copolymers of various polysaccharides containing $\mathrm{P}-\mathrm{Hyd}-$ PAM had a wide range of compressive strength and the water absorbencies from $40 \mathrm{~g}$ to $210 \mathrm{~g}$ and $390 \mathrm{~g} / \mathrm{g}$ to $3000 \mathrm{~g} / \mathrm{g}$, respectively. These results suggest that the water absorbency and the compressive strength of the graft copolymers of $\mathrm{HEC}$ and the other polysac. charides especially depend on the kinds of branch and trunk polymers.

\subsection{Effects of $\mathrm{pH}$, Temperature and Aqueous Solvent Composition on Absorbency}

Fig. 8 shows that the water absorbency of the HEC graft copolymers and a commercial super waterabsorbent varied with $\mathrm{pH}$. A sharp maximum appeared at $\mathrm{pH} 7$, and beyond this $\mathrm{pH}$, the water absorbency decreased and reached a low value at $\mathrm{pH}$ 13. When the $\mathrm{pH}$ is reduced, some of the ionized carboxyl groups on the HEC graft copolymers recombine

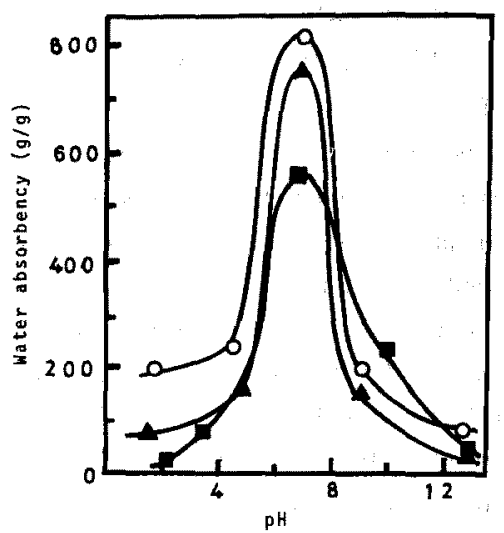

Fig. 8. Water absorbency versus pH. O. HEC-P-HydPAM (grafting 507.1\%; hydrolysis $65.9 \%$ ); $\boldsymbol{A}$, HECP-Hyd-PMA (hydrolysis $75.6 \%$ ); . Commercial waterabsorbent (Sanwet).

with super-abundant hydrogen ions. As a result, the water absorbency is reduced at acidic $\mathrm{pH}$. When the $\mathrm{pH}$ is raised, the concentrations of sodium and hy. droxide ions increase in the solution, and then the osmotic pressure and the polymer-solvent affinity decrease. Therefore, the water absorbency is reduced. It is known that the super water-absorbents with carboxyl groups have a weakpoint in the $\mathrm{pH}$ dependence of water absorbency [2]. The HEC graft copolymers as super water-absorbents could not overcome this weakpoint.

It is reported[7] that partially hydrolyzed PAM gels absorb a lot of water, and they swell at temperatures higher than room temperature and shrink below

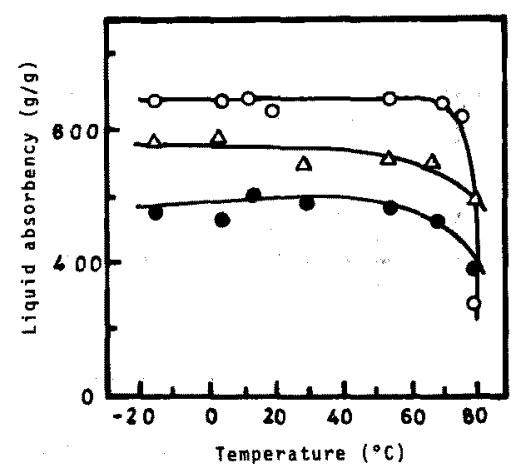

Fig. 9. Effect of temperature on the liquid absorbency of HEC-P-Hyd-PAM (grafting 507.1\%; hydrolysis $65.9 \%$ ). Liquid: $O$, water: $\Delta$, water: methanol $(60: 40)$; and water: methanol $(40: 60)$. 


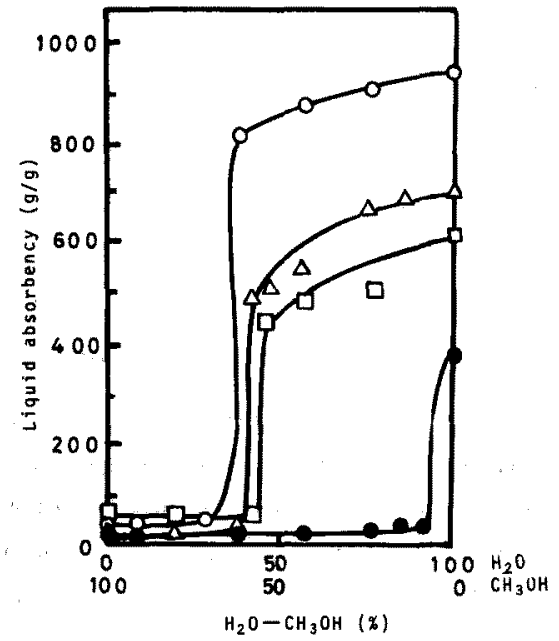

Fig. 10. Effects of the degree of hydrolysis and the composition of aqueous methanol solution on the liquid absorbency of HEC-P-Hyd-PAM (grafting 615.8\%). Degree of hydrolysis: $\bigcirc, 88.1 \% ; \triangle, 74.6 \% ; \square, 66.0 \%$; and $11.4 \%$.

room temperature. However, the liquid absorbency of HEC-P-Hyd-PAM was independent of the temperature in the range from $-20^{\circ} \mathrm{C}$ to $70^{\circ} \mathrm{C}$, as shown in Fig. 9.

Fig. 10 shows the effect of the composition of aqueous methanol solutions on the liquid absorbency of HEC-P-Hyd-PAM. The liquid absorbency was high at low methanol concentrations, and as the methanol concentration increased, it gradually decreased. The liquid absorbency steeply decreased by a factor of 40 at about $55-70 \%$ methanol concentration, and remained low at higher concentrations of methanol. Partially hydrolyzed PAM gels are found $[9]$ to undergo discrete volume transitions upon changes in solvent composition, $\mathrm{pH}$, salt concentration of the solution or in temperature. It was found that
HEC-P-Hyd-PAM underwent also similar discrete volume transitions upon changing the $\mathrm{CH}_{3} \mathrm{OH}-\mathrm{H}_{2} \mathrm{O}$ composition. These steep changes in the fluid absorbency of HEC-P-Hyd-PAM due to the change in the solvent composition are based on three forces; the osmotic pressure which is associated with the ionization of the absorbent network, the polymer-solvent affinity, and the rubber elasticity based on the resist. ance of the individual polymer chains to stretching or compression. The total force acting on the HEC water absorbents is the sum of the above mentioned forces. The competition among the three forces changes, the fluid absorbency of HEC-P-Hyd-PAM.

\section{REFERENCES}

1. G. F. Fanta, R. C. Burr, C. R. Russel, and C. E. Rist, J. Appl. Polym. Sci., 11, 457 (1967); U. S. P. 3,981,100-1976

2. T. Motohashi, Kobunshi Kako, 33, 452 (1984)

3. P. Lepoutre, S. H. Hui, and A. A. Robertson, J. Macromol. Sci-Chem., A-10, 681 (1976)

4. N. Miyata and I. Sakata, "Wood and Cellulosies", (J. F. Kennedy, G. O. Phillips, D. J. Wedlock, and P. A. Williams eds.), Ellis Horwood, Chichester, U. K. p. 491 (1987)

5. G. Mino and S. Kaizerman, J. Polym. Sci, 31, $242(1958)$

6. P. J. Flory "Principles of Polymer Chemistry", Cornell University Press, Ithaca, U. S. A., p. 529 (1965)

7. T. Tanaka, Polymer, 20, 1404 (1979); D. Nicoli, C. Young, T. Tanaka, A. Pollak, and G. Whitesides, Macromolecules, 16, 887 (1983)

8. I. Murase and F. Fujita, Kogyo Zairyo, 29, [8], 34 (1981)

9. T. Tanaka, I. Nishio, S. t. Sun, and S. U. Nishio, Science, 218, 467 (1982) 